\title{
COMPARISON OF CLINICAL AND LABORATORY CHARACTERISTICS OF PATIENTS WITHEARLY VS. LATE ONSET PRIMARY ANTIPHOSPHOLIPID SYNDROME
}

Carla Baleeiro Rodrigues Silva ${ }^{1, *}$, Camila da Silva Cendon Duran ${ }^{1}$, Flavio Signorelli ${ }^{1,2}$, Gustavo Guimarães Moreira Balbi ${ }^{1,3}$, Eloisa Silva Dutra de Oliveira Bonfá ${ }^{1}$, Danieli Castro de Oliveira Andrade ${ }^{1}$

1. Universidade de São Paulo, São Paulo (SP), Brazil. 2. Universidade do Estado do Rio de Janeiro, Rio de Janeiro (RJ), Brazil. 3. Universidade Federal de Juiz de Fora, Juiz de Fora (MG), Brazil.

*Corresponding author: caca.baleeiro@gmail.com

\section{BACKGROUND}

Antiphospholipid syndrome (APS) is the most common cause of acquired thrombophilia. Clinical manifestations include arterial and/ or venous thromboses and pregnancy morbidity in the presence of antiphospholipid antibodies. Some studies suggest that children with APS have a higher risk of thromboembolic events than adults, and patients who developed APS later in life present with a higher frequency of lupus anticoagulant, triple positivity to antiphospholipid antibodies and arterial thrombosis. Nonetheless, studies describing the clinical and laboratory course of the disease among patients with APS onset in youth (15-24 years) are scarce. The aim of this study is to compare clinical and laboratory characteristics of patients with early (24 years old or less) vs. late (over 24 years old) onset APS.

\section{MATERIALS AND METHODS}

A cross-sectional analysis was performed with 250 primary APS patients (PAPS) regularly followed in the APS outpatient clinics from two centers (ICHC/USP and HUPE/UERJ). Data retrieval (demographics, criteria and noncriteria manifestations, cardiovascular risk factors and aPL status) was performed during visits and by chart review. The patients were then divided into two groups, based on the age at presentation: patients with age at presentation between 15 and 24 years were classified as early onset, and those with age at presentation over 24 years were classified as late onset. Both groups were compared regarding the aforementioned characteristics. Statistical analysis was performed using chi-square, Mann-Whitney $U$ and t test, when applicable.

\section{RESULTS}

Of the 250 patients included, 76 had early onset and 174 late onset APS. The cohort overall characteristics is summarized in Table 1. The PAPS duration was similar between groups ( $16.5 \pm 10.8$ years in the early onset group vs. $14.7 \pm 9.6$ years in the late onset group; $p=0.195$ ). The aPL status was also similar between early and late onset patient. Site of first manifestation: Obstetric events were more frequently observed in the early onset group ( $31.6 \%$ vs. $14.5 \%, p=0.002$ ), as opposed to thrombotic events in the late onset group ( $85.5 \%$ vs. $68.4 \% \mathrm{p}=0.002)$. The number of pregnancies was similar between groups $(2.8 \pm 2.5$ in the early onset group vs. $2.7 \pm 2.1$ in the late onset group; $p=0.899$ ). Obstetric events were more common in the early onset group - fetal death $(40.9 \%$ vs. $27.0 \%, p=0.009)$ and preterm delivery $(25.0 \%$ vs. $11.5 \%, p=0.003)$. Noncriteria manifestations: Skin ulcers were also more frequent in the early onset group ( $17.1 \%$ vs. $4.0 \%, p=0.001)$. Cardiovascular risk factors: hypertension ( $45.4 \%$ vs. $25.0 \%$, $p=0.002$ ), dyslipidemia (48.3\% vs. $30.3 \%, p=0.008$ ), nephropathy ( $8 \%$ vs. $1.3 \%, p=0.039$ ), and smoking ( $37.4 \%$ vs. $18.4 \%, p=$ 0.003 ) were more common in patients with late onset APS.

\section{CONCLUSION}

Obstetric manifestations, both as initial presentation or throughout follow-up, are more frequently seen in early onset PAPS. Since older patients are more likely to present cardiovascular risk factors, this finding may explain why they are more likely to present with a thrombotic event. Longitudinal studies are needed to confirm these observations. 
Table 1. Criteria and non-criteria characteristics, obstetric manifestations and aPL profile in 76 young-and 174 adult-onset primary APS patients.

\begin{tabular}{|c|c|c|c|}
\hline Thrombotic manifestations & Young onset $\mathrm{N}=76$ & Adult Onset $\mathrm{N}=174$ & $\mathrm{p}$ \\
\hline Arterial thrombosis & $26(34.2 \%)$ & $73(42.0 \%)$ & 0.250 \\
\hline Recorrent arterial event & $8(30.8 \%)$ & $34(46.6 \%)$ & 0.161 \\
\hline Stroke/TIA & $18(23.7 \%)$ & $48(27.6 \%)$ & 0.520 \\
\hline Myocardial infarction & $3(3.9 \%)$ & $11(6.3 \%)$ & 0.453 \\
\hline Venous thrombosis & $53(43.4 \%)$ & $121(69.5 \%)$ & 0.975 \\
\hline Recurrent venous event & $23(43.4 \%)$ & $49(40.8 \%)$ & 0.753 \\
\hline Small vessel thrombosis & $5(6.6 \%)$ & $12(6.9 \%)$ & 0.927 \\
\hline Catastrophic syndrome & $0(0.0 \%)$ & $1(0.6 \%)$ & 0.508 \\
\hline Non-criteria manifestations & Young $\mathrm{N}=76$ & Adult Onset N=174 & $\mathrm{p}$ \\
\hline Migraine & $36(47.4 \%)$ & $68(39.1 \%)$ & 0.252 \\
\hline Livedo reticularis & $25(32.9 \%)$ & $58(33.3 \%)$ & 0.905 \\
\hline Raynaud's phenomenon & $22(28.9 \%)$ & $57(32.8 \%)$ & 0.521 \\
\hline Skin ulcer & $13(17.1 \%)$ & $7(4.0 \%)$ & 0.001 \\
\hline Valvulopathy & $9(17.0 \%)$ & $24(18.9 \%)$ & 0.762 \\
\hline Convulsion & $8(10.5 \%)$ & $14(8.0 \%)$ & 0.524 \\
\hline Thrombocytopenia & $6(7.9 \%)$ & $26(14.9 \%)$ & 0.125 \\
\hline Hemolytic anemia & $6(7.9 \%)$ & $2(1.1 \%)$ & 0.146 \\
\hline Chorea & $2(2.6 \%)$ & $2(1.1 \%)$ & 0.390 \\
\hline Multiple Sclerosis like & $0(0.0 \%)$ & $4(2.3 \%)$ & 0.183 \\
\hline Obstetric manifestations & Young $N=53$ & Adult Onset N=174 & $\mathrm{p}$ \\
\hline Fetal death (>10 weeks) & $31(58.5 \%)$ & $47(37.3 \%)$ & 0.009 \\
\hline Early miscarriage ( $<10$ weeks) & $24(45.3 \%)$ & $47(37.3 \%)$ & 0.319 \\
\hline Premature birth & $19(35.8 \%)$ & $20(15.9 \%)$ & 0.003 \\
\hline Recurrent early miscarriage & $4(7.5 \%)$ & $15(12.2 \%)$ & 0.362 \\
\hline aPL profile & Young $\mathrm{N}=76$ & Adult Onset N=174 & $\mathrm{p}$ \\
\hline Lupic anticoagulant + & $71(93.4 \%)$ & $164(94.3 \%)$ & 0.897 \\
\hline Anticardiolipin + & $38(50.0 \%)$ & $82(47 . \%)$ & 0.676 \\
\hline Anticardiolipin $>80$ & $20(26.3 \%)$ & $57(32.8 \%)$ & 0.304 \\
\hline Anti-ß2-GPI + & $32(42.1 \%)$ & $83(47.7 \%)$ & 0.355 \\
\hline Triple-positive & $19(25.0 \%)$ & $52(29.9 \%)$ & 0.478 \\
\hline
\end{tabular}

